\title{
Ligand Addition Energies and the Stoichiometry of Colloidal Nanocrystals
}

\author{
Michael Sluydts, $^{\dagger}$ Kim De Nolf, ${ }^{\ddagger} \uparrow$ Veronique Van Speybroeck, ${ }^{\dagger}$ Stefaan \\ Cottenier, ${ }^{*, \dagger}$ and Zeger Hens, $*,+\uparrow$ \\ $\dagger$ Center for Molecular Modeling, Ghent University, Belgium \\ $\ddagger$ Physics and Chemistry of Nanostructures, Ghent University, Belgium \\ ฯ Center for Nano and Biophotonics, Ghent University, Belgium \\ $\S$ Department of Materials Science and Engineering, Ghent University, Belgium \\ E-mail: stefaan.cottenier@ugent.be; zeger.hens@ugent.be
}

\section{S1 Excess surface of $\mathrm{ZnSe}$ and InP}
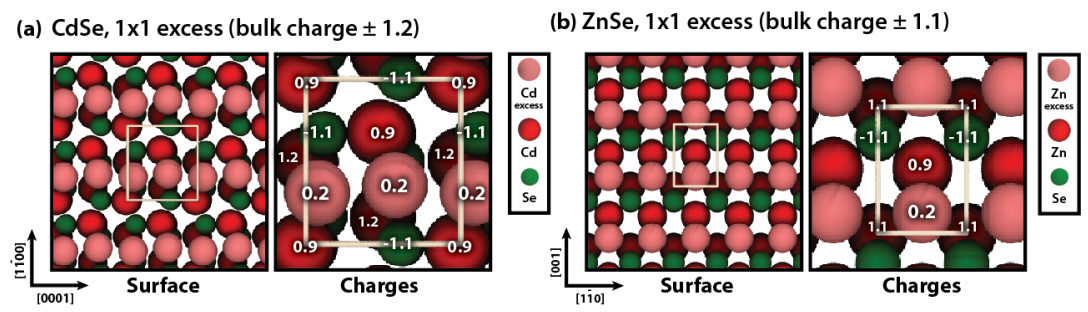

Figure S1: Top view of the excess surfaces in their unit cells (a) CdSe cell (b) ZnSe (c) InP

Figure S1 shows the excess surfaces of the CdSe and ZnSe unit cells together with their Hirshfeld-I charges. The discussion of CdSe can be found in the main text. CdSe and ZnSe have effectively the same charges, but arranged in a different lattice. The only difference can be found in the bulk charges which differ slightly ( \pm 1.2 for CdSe and \pm 1.1 for ZnSe). Figure S2 shows the $1 \times 1$ unit cell and the two studied supercells of InP. 
(a) $\ln P, 1 \times 1$ excess (bulk charge \pm 1.4 )

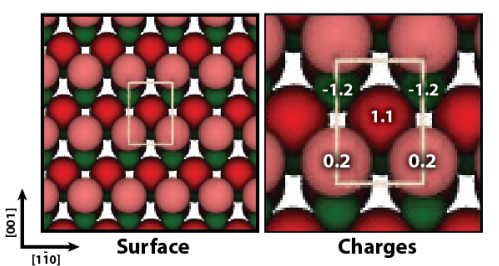

(b) $\ln P, 2 \times 1$ excess (bulk charge \pm 1.4 )

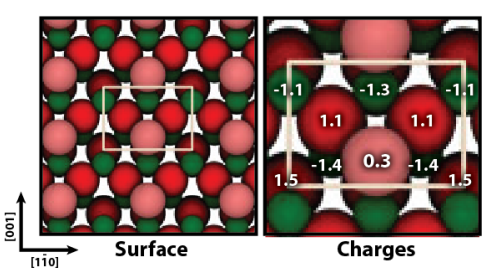

(c) $\ln P, 1 \times 2$ excess (bulk charge \pm 1.4 )

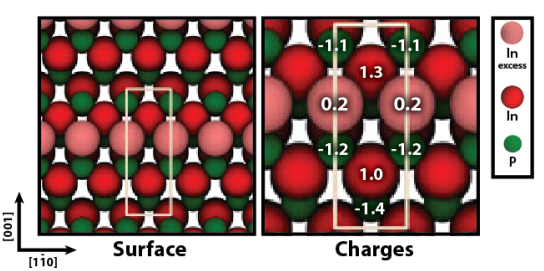

Figure S2: Top view of the excess InP surface (a) $1 \times 1$ cell (b) $2 \times 1$ cell (c) $1 \times 2$ cell.

The bulk charges here are slightly larger $( \pm 1.4)$, corresponding to the higher effective charges of In and $\mathrm{P}( \pm 3)$. In the unit cell this leads to a greater surface charge for both In $(+1.1)$ and $\mathrm{P}(-1.2)$ while the excess atom charge remains +0.2 . To create the supercells excess atoms are removed, leading to inequivalent surface atoms. The $\mathrm{P}$ atom in contact with the excess atom has a larger charge of $-1.3(2 \times 1$ cell $)$ or $-1.2(1 \times 2$ cell $)$ having transferred charge to the excess atom, while the free $\mathrm{P}$ has a charge of -1.1. The $\mathrm{P}$ atoms found deeper in the slab converge to their bulk values of -1.4. The In atoms follow a similar behavior, showing a higher charge when higher-coordinated. This gives the surface In a +1.1 charge in the $2 \times 1$ cell and a +1.3 charge in the $1 \times 2$ cell. The bulk charge can go up to +1.5 representing a charge transfer to the center of the slab. This is due to the accumulation of charge from the outer layers, retreating from the vacuum.

\section{S2 Ligand reactions}

The main text already showed several interactions between ligand molecules which were mainly limited to the formation of dimers. In the case of hydrochalcogens the ligand can also be dehydrogenated with the $\mathrm{H}$ atom being transferred to nearby ligands. An example of such processes is shown in Figure S3.

Figure S3a shows the dehydrogenation of a SeH ligand with the resulting Se atom embedding itself in the bulk, forming a stoichiometric CdSe surface. The H atom transfers itself to the second $\mathrm{SeH}$ ligand, forming a $\mathrm{SeH}_{2}$ molecule which lightly coordinates with the surface through $\mathrm{H}$ bonding. Figure S3b shows the dimerization of SH ligands, resulting in 

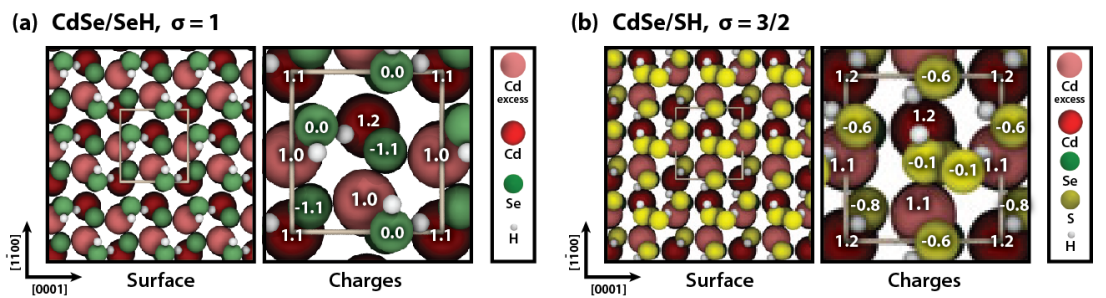

Figure S3: Ligand reactions on the nanocrystal surface: (a) $2 \mathrm{ZH} \rightarrow \mathrm{Z}+\mathrm{ZH}_{2}$ ( $\mathrm{H}$ charge $+0.07-0.08)(\mathrm{b}) 2 \mathrm{ZH} \rightarrow \mathrm{Z}_{2} \mathrm{H}_{2}(\mathrm{H}$ charge $+0.07-0.12(\mathrm{~mol})+0.2($ surf $))$.

a detached $\mathrm{S}_{2} \mathrm{H}_{2}$ molecule. These reactions occurred spontaneously during the relaxation process. The kinetics of the process were not further studied.

\section{S3 Suprastoichiometric adsorption of oxygen on InP}

The $\mathrm{InP} / \mathrm{O}$ system was discussed in detail in the main text up to $\sigma=2$. Figures $\mathrm{S} 4 \mathrm{a}$ and $\mathrm{b}$ repeat the $\sigma=4 / 3$ and $\sigma=2$ which took place in the $2 \times 1$ supercell. Figure $\mathrm{S} 4 \mathrm{c}$ shows one additional step which is possible with an addition energy of approximately $0 \mathrm{~kJ} / \mathrm{mol}$ without detachment of the excess complex.
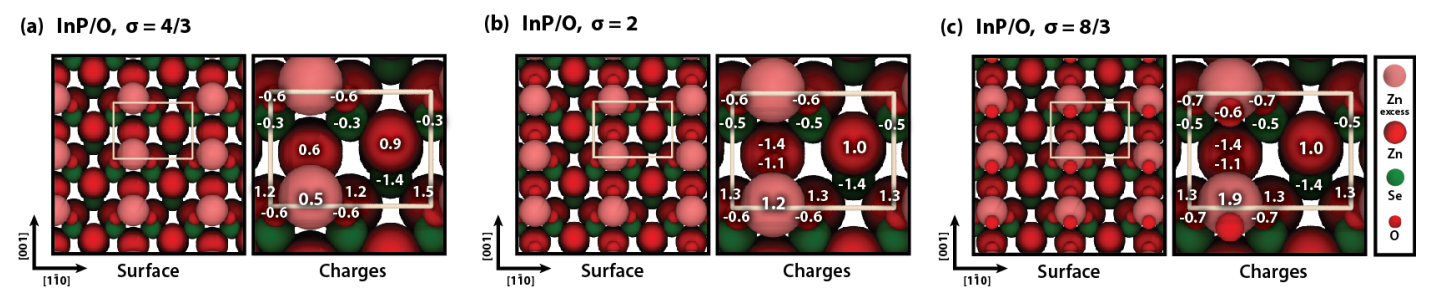

Figure S4: Top view on the InP (110) surface subjected to progressive oxygen addition with occupation increasing from (a) $4 / 3$ to (b) 2 and (c) $8 / 3$.

This additional $\mathrm{O}$ atom adsorbs on top of the excess In, leading to a fourfold coordination of this atom by $\mathrm{O}$, each with a Hirschfeld-I charge of -0.6 to -0.7 . The charge on the excess atom increases up to +1.9 , a value far above the bulk value of +1.4 . This illustrates the strong tendency of the $\mathrm{InP}$ crystal to be oxidated with respect to the reference state of $\mathrm{O}_{2}$ and $\mathrm{H}_{2} \mathrm{O}$. 


\section{S4 Ligand addition energies}

Figure S5 repeats the ligand addition energies previously shown in Figure 4 of the main text. Results are shown for each combination of the three materials (CdSe, ZnSe and InP) and the three ligand classes (chalcogens, hydrochalcogens and halogens).
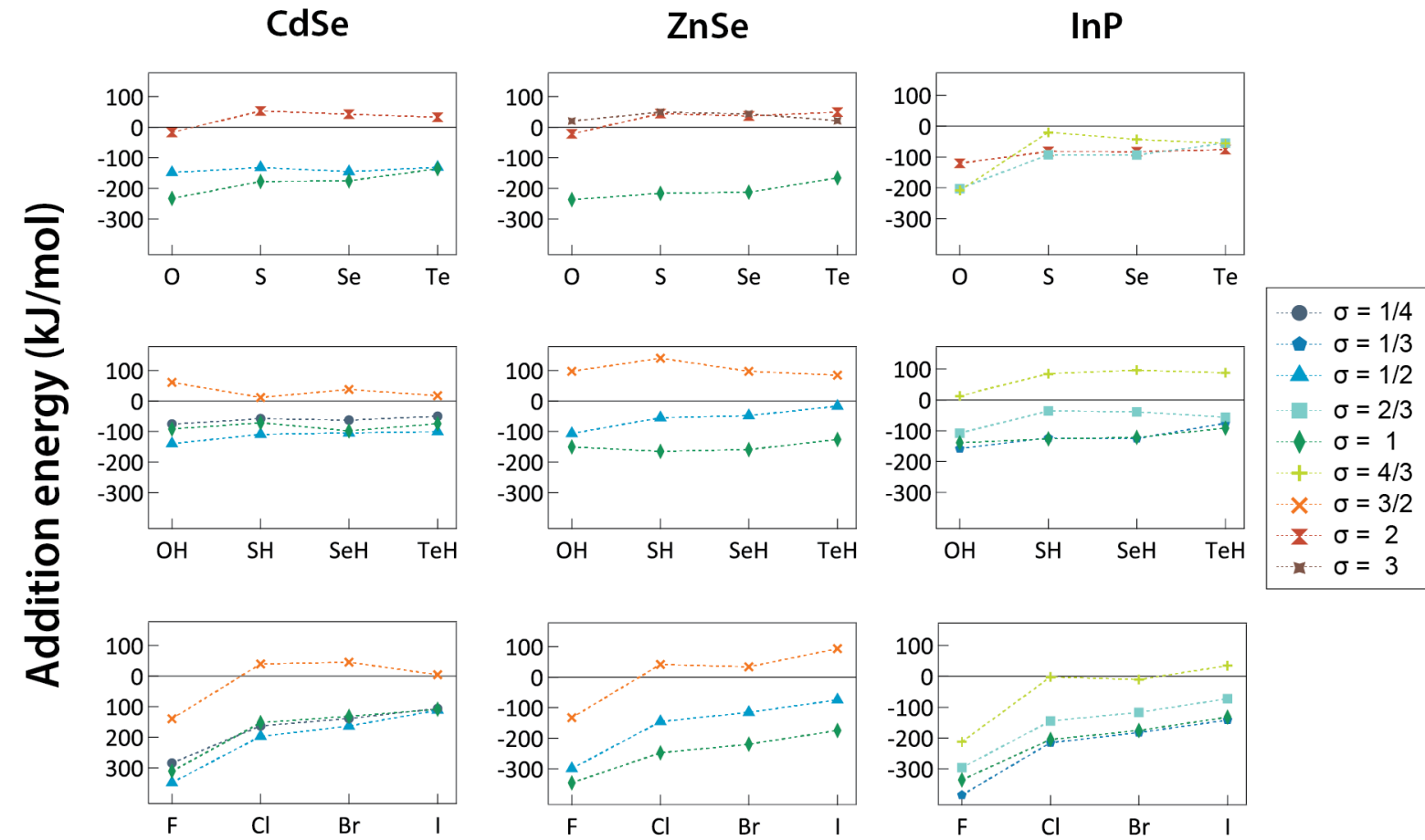

Figure S5: Ligand addition energy (in $\mathrm{kJ} / \mathrm{mol}$ ) for the different combinations of ligand set and material. Rows corresponds to the ligand family while materials are represented by the columns. Negative addition energies correspond to adsorbed states. Stoichiometry ratio varies by color with yellow to red corresponding to sub- and superstoichiometric.

\section{S5 Surface geometries of the stable surfaces}

In this section the top view of each surface corresponding to the data points plotted in Figure S5 is shown. Each individual figure corresponds to one combination of material and ligand family and thus one of the Figure S5 subplots. CdSe surface can be found in Figures S6, S7 and S8, ZnSe in Figures S9, S10 and S11 and InP in Figures S12, S13 and S14. Each of the groups starts with the chalcogens, followed by hydrochalcogens and finally halogens. 

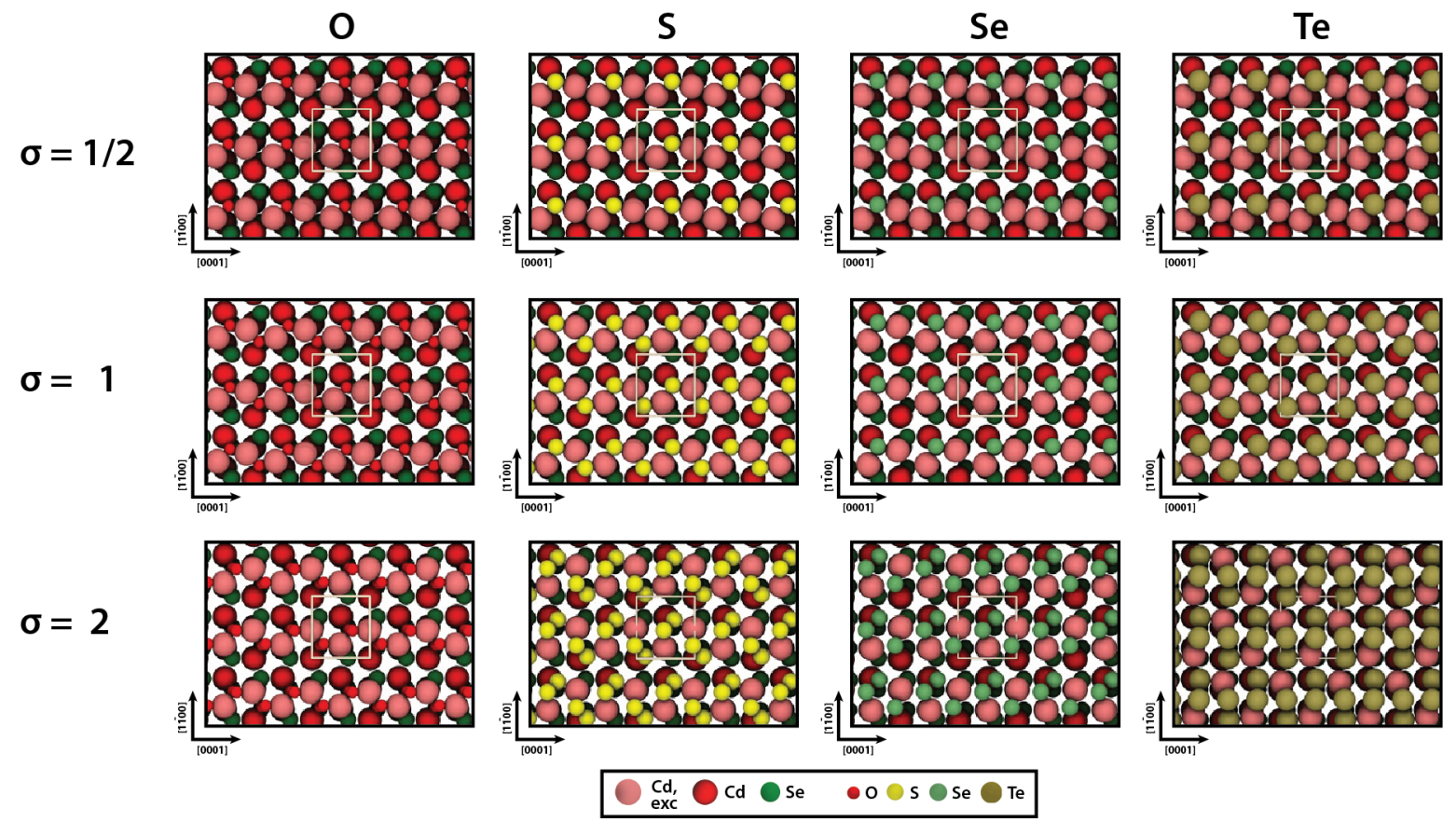

Figure S6: Most stable chalcogen-adsorbed CdSe surfaces. Ligand species varies from left to right: $\mathrm{O}, \mathrm{S}, \mathrm{Se}$ and Te. Stoichiometric ratio varies from top to bottom: $\sigma=1 / 2,1$ and 2 . 


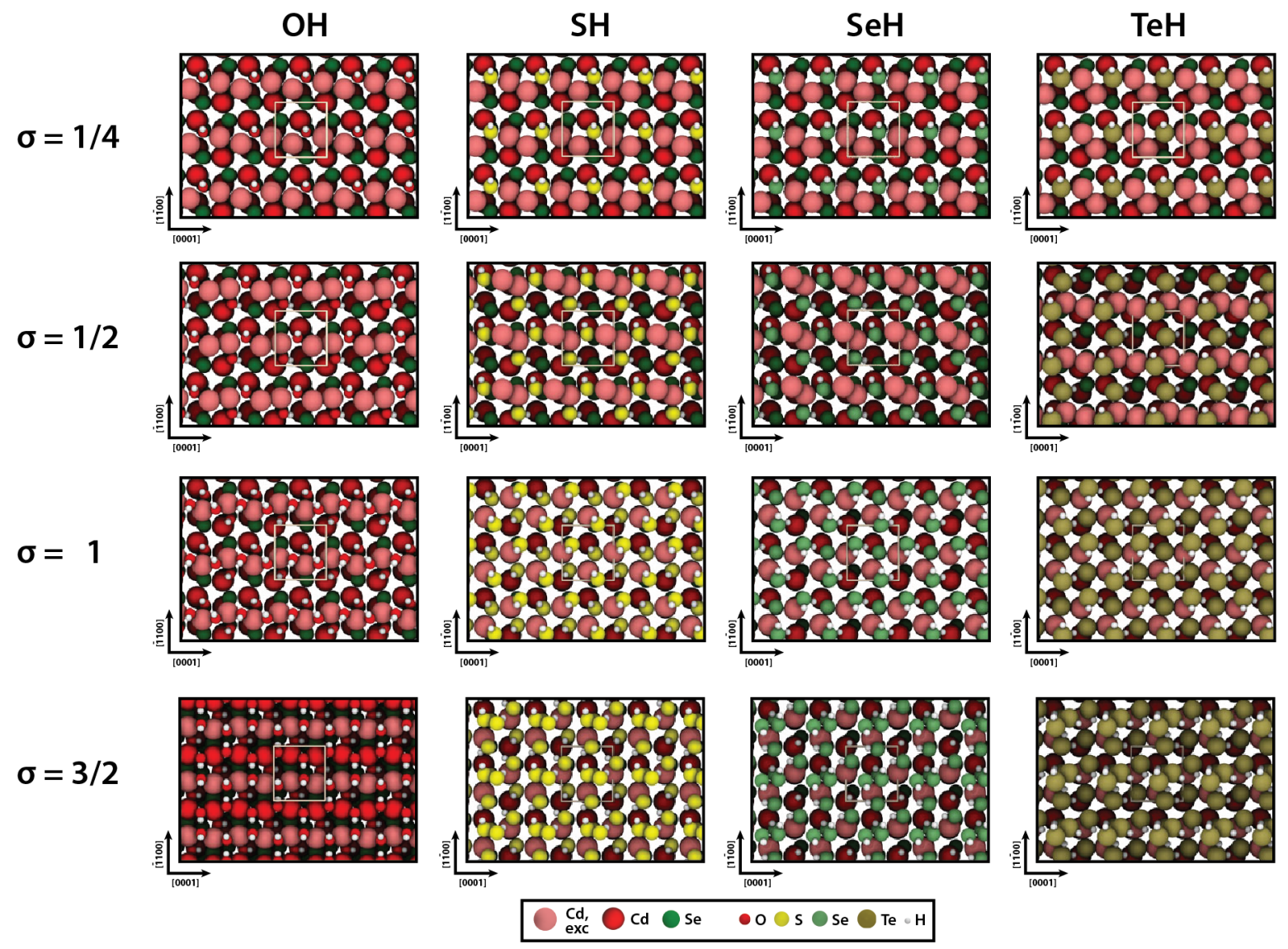

Figure S7: Most stable hydrochalcogen-adsorbed CdSe surfaces. Ligand species varies from left to right: $\mathrm{OH}, \mathrm{SH}, \mathrm{SeH}$ and TeH. Stoichiometric ratio varies from top to bottom: $\sigma=$ $1 / 4,1 / 2,1$ and $3 / 2$. 

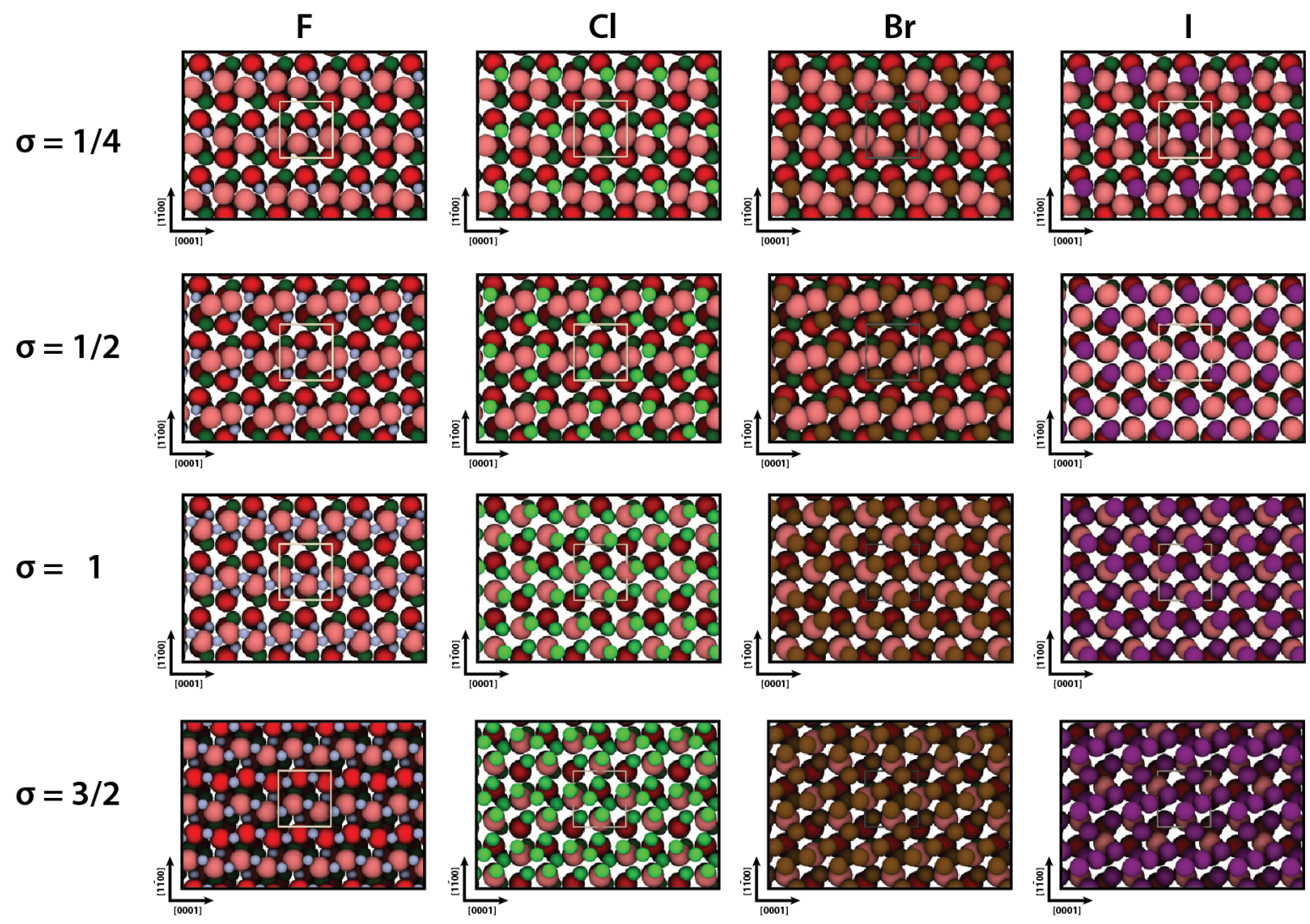

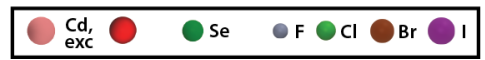

Figure S8: Most stable halogen-adsorbed CdSe surfaces. Ligand species varies from left to right: $\mathrm{F}, \mathrm{Cl}, \mathrm{Br}, \mathrm{I}$. Stoichiometric ratio varies from top to bottom: $\sigma=1 / 4,1 / 2,1$ and 3/2. 

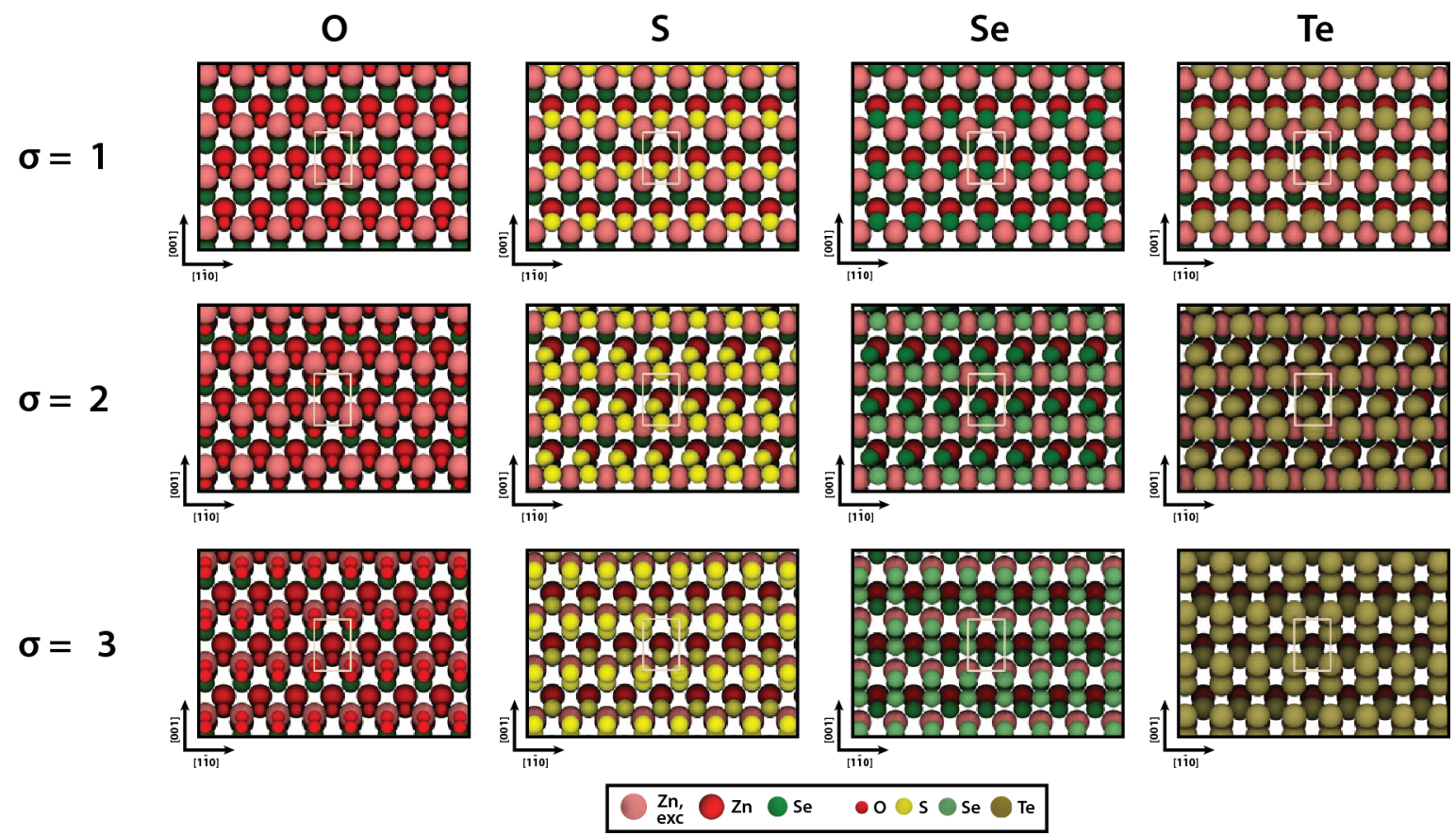

Figure S9: Most stable chalcogen-adsorbed ZnSe surfaces. Ligand species varies from left to right: O, S, Se and Te. Stoichiometric ratio varies from top to bottom: $\sigma=1,2$ and 3 .
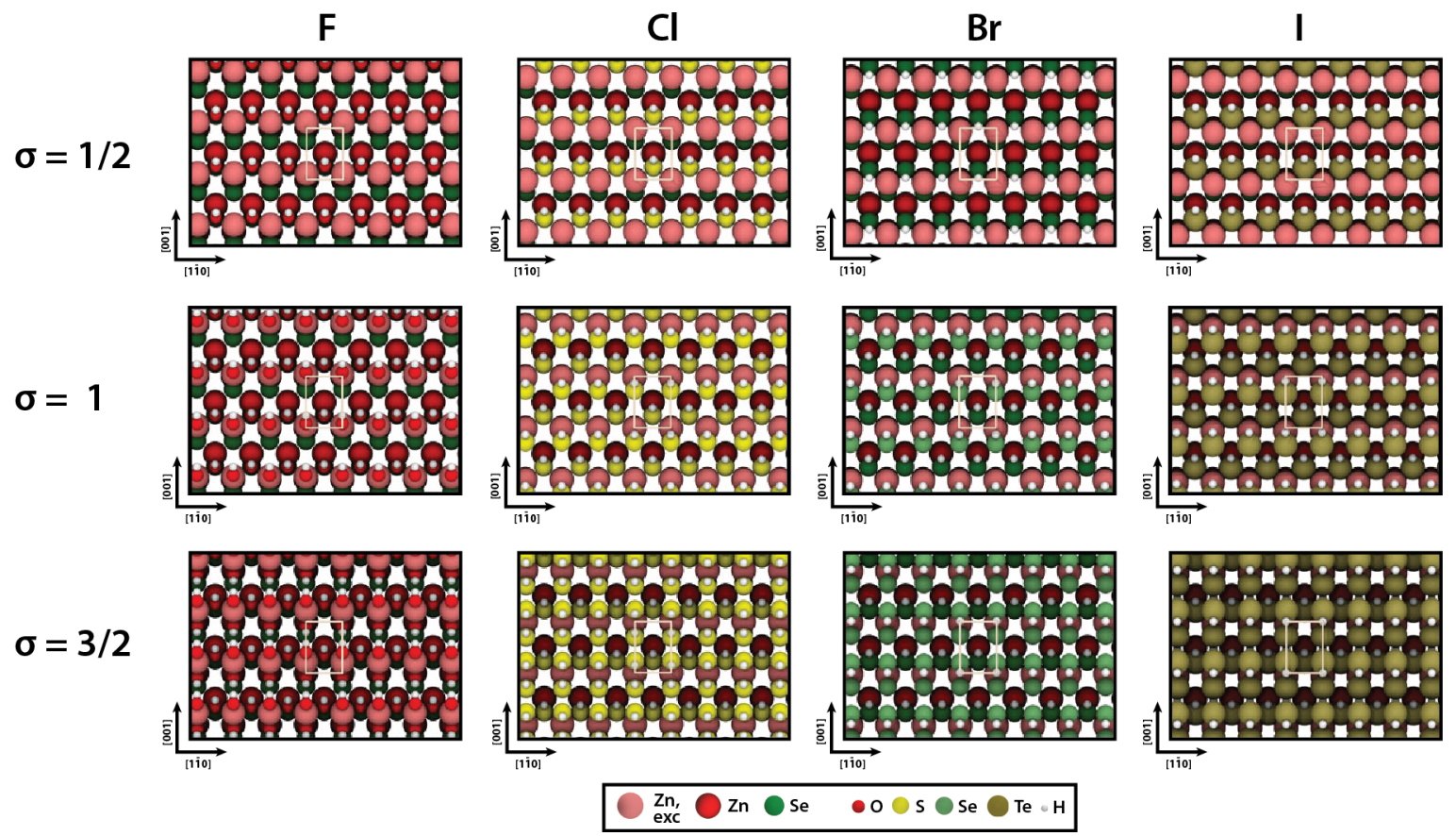

Figure S10: Most stable hydrochalcogen-adsorbed ZnSe surfaces. Ligand species varies from left to right: $\mathrm{OH}, \mathrm{SH}, \mathrm{SeH}$ and TeH. Stoichiometric ratio varies from top to bottom: $\sigma=$ $1 / 2,1$ and $3 / 2$. 


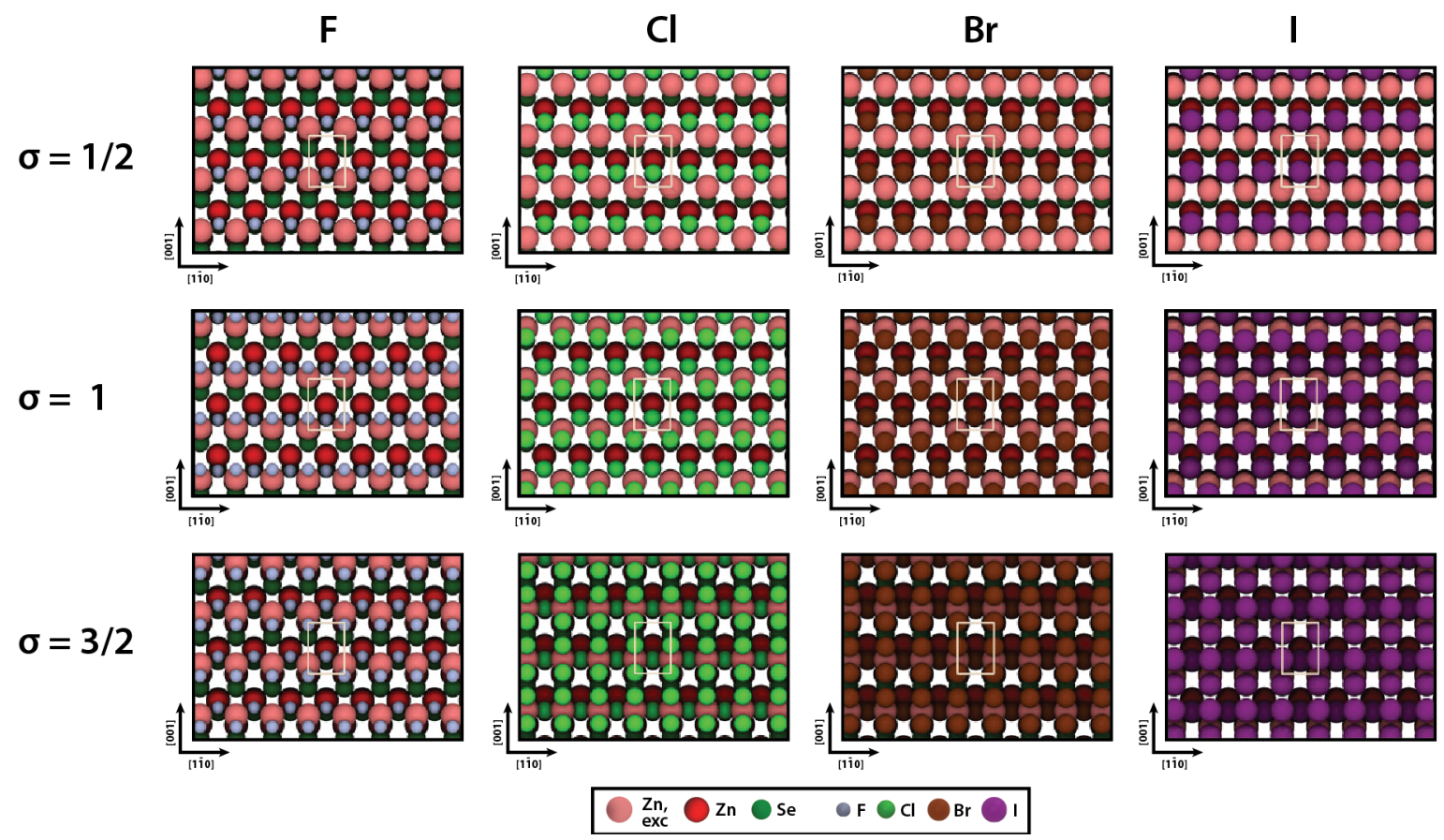

Figure S11: Most stable halogen-adsorbed ZnSe surfaces. Ligand species varies from left to right: F, Cl, Br, I. Stoichiometric ratio varies from top to bottom: $\sigma=1 / 2,1$ and $3 / 2$.

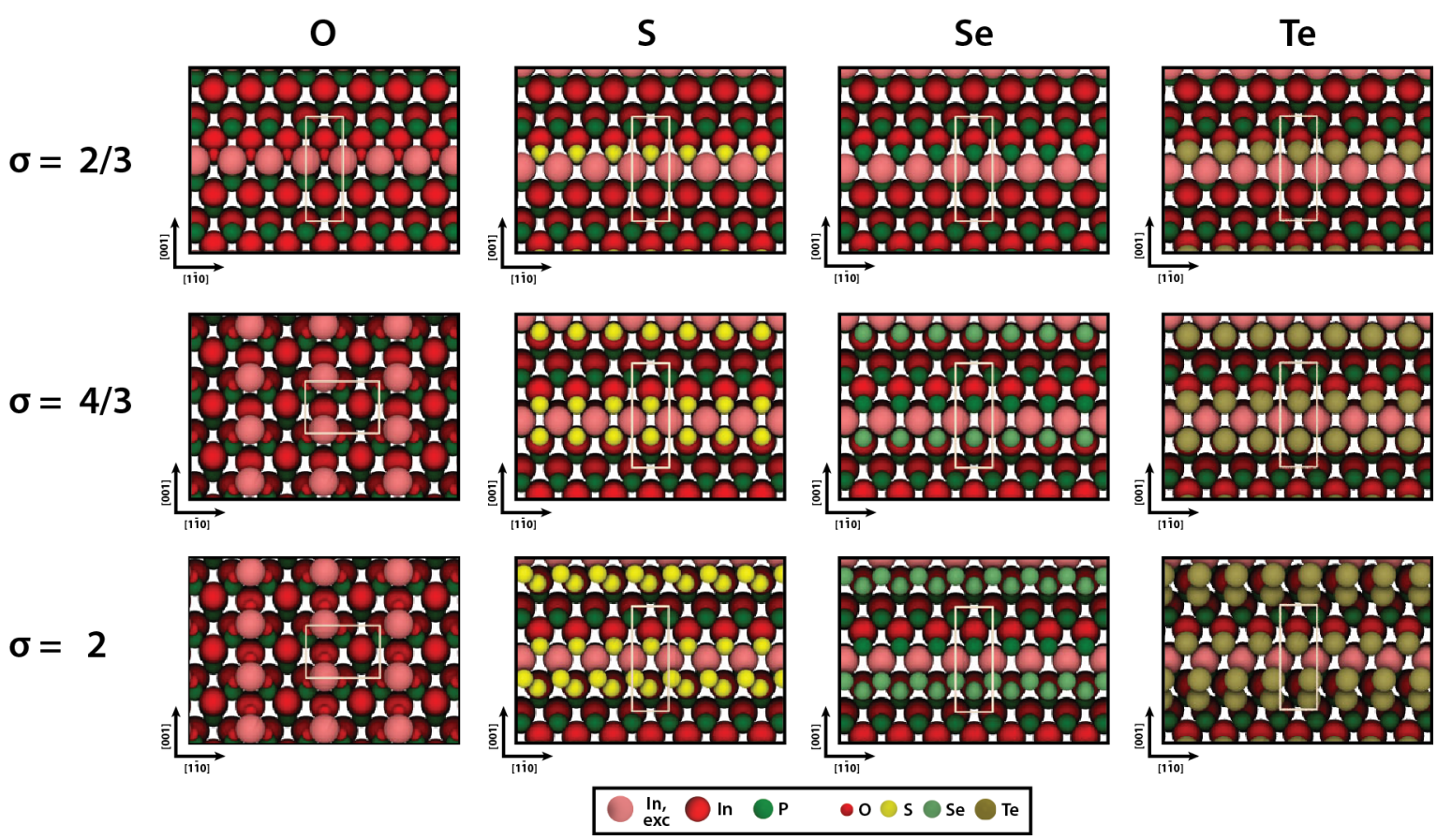

Figure S12: Most stable chalcogen-adsorbed InP surfaces. Ligand species varies from left to right: O, S, Se and Te. Stoichiometric ratio varies from top to bottom: $\sigma=2 / 3,4 / 3$ and 2. 


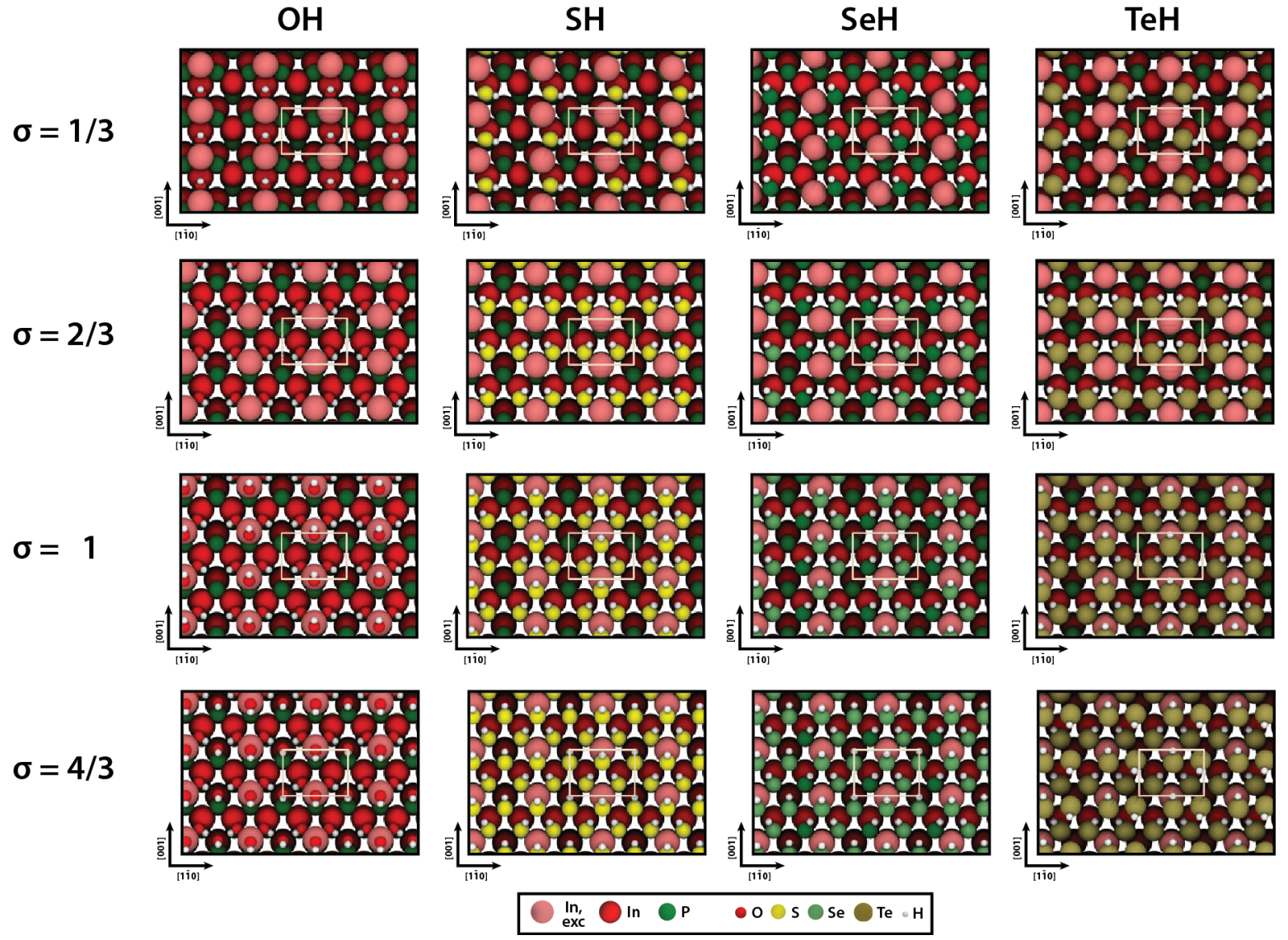

Figure S13: Most stable hydrochalcogen-adsorbed InP surfaces. Ligand species varies from left to right: $\mathrm{OH}, \mathrm{SH}, \mathrm{SeH}$ and $\mathrm{TeH}$. Stoichiometric ratio varies from top to bottom: $\sigma=$ $1 / 3,2 / 3,1$ and $4 / 3$. 


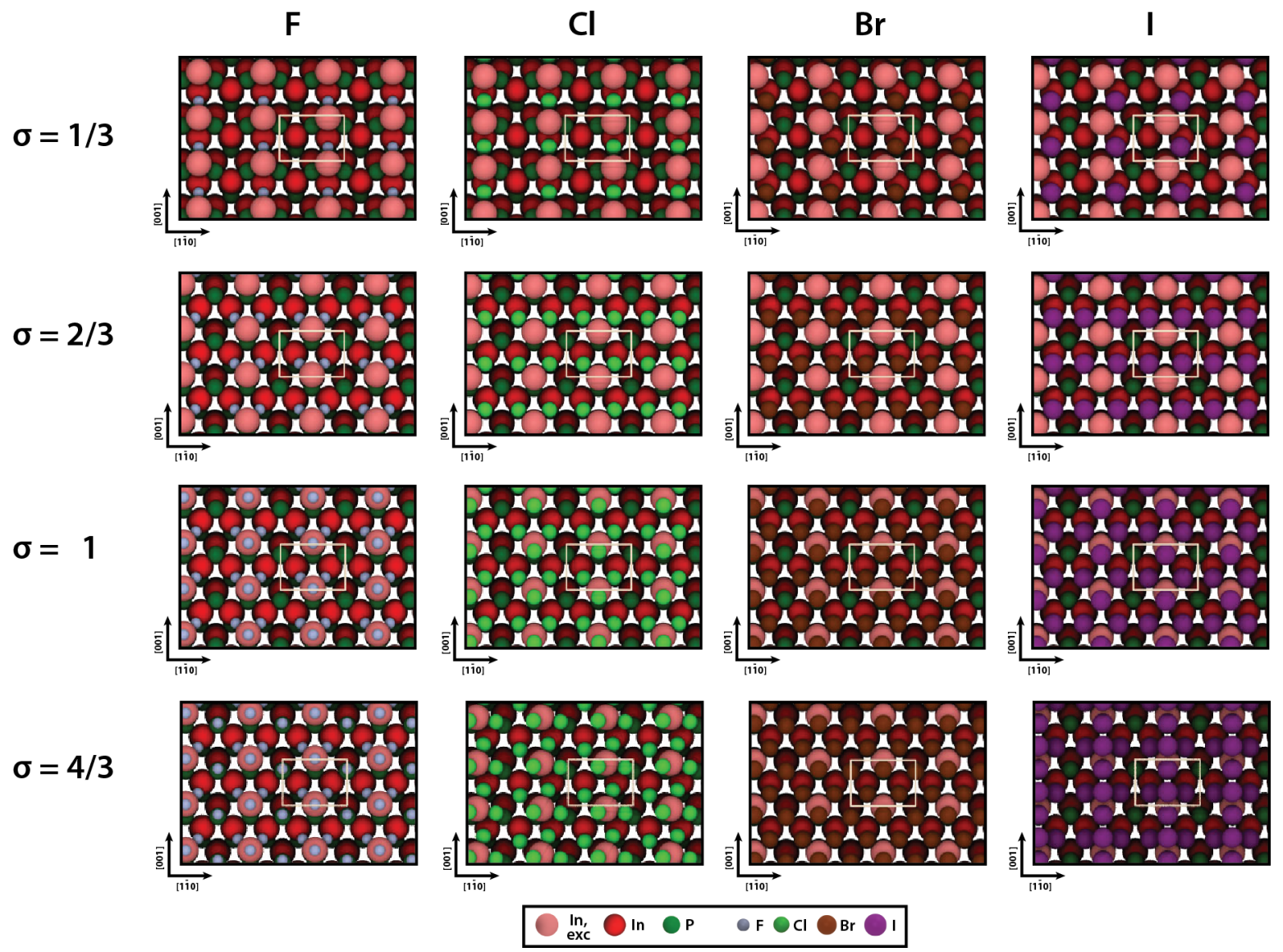

Figure S14: Most stable halogen-adsorbed InP surfaces. Ligand species varies from left to right: F, Cl, Br, I. Stoichiometric ratio varies from top to bottom: $\sigma=1 / 3,2 / 3,1$ and $4 / 3$. 